Trauma Berufskrankh 2008 10 [Suppl 2]:165-166

DOI 10.1007/s10039-008-1363-3

Online publiziert: 19. April 2008

(c) Springer Medizin Verlag 2008

\author{
J. Freyschmidt \\ Beratungsstelle und Referenzzentrum für Osteoradiologie, \\ Klinikum Bremen Mitte, Bremen
}

\title{
Die pathologische Fraktur aus Sicht des Radiologen
}

delt es sich um eine Korrelation zwischen der Wachstumsgeschwindigkeit einer osteolytischen Läsion einerseits und der radiologischen Morphologie.

Man unterscheidet 3 Grade, wobei die Grade I und II für eine geografische, d. h. zusammenhängende Osteolyse eingesetzt werden und der Grad III für eine diskontinuierliche und nicht zusammenhängende - mottenfraßartige - Läsion steht. Grad I wird in die Untergruppen A-C eingeteilt. Grad IA entspricht der langsamsten Wachstumsgeschwindigkeit, man findet immer einen Sklerosesaum. Meist handelt es sich um benigne Läsionen wie das nichtossifizierende Knochenfibrom oder auch eine fibröse Dysplasie. Beim Grad IB, der eine höhere Wachstumsgeschwindigkeit als Grad IA hat, findet sich die originäre Kortikalis abgebaut und durch eine neue Schale ersetzt (z. B. bei der einkammerigen juvenilen Knochenzyste), beim Grad IC ist die Kortikalis an einer Stelle immer perforiert, was insbesondere bei Riesenzelltumoren zutrifft. Die nächsthöhere Wachstumsgeschwindigkeit entspricht dem Grad II, sie hat als Ausdruck ihres sehr aggressiven Wachstums einen mottenfraßartigen Rand. Ihm bzw. Grad III entsprechen die meisten Osteo-, Ewing- sowie Fibrosarkome, aber auch akute Osteomyelitiden. Grad III hat die höchste Wachstumsgeschwindigkeit und ist durch diskontinuierliche „mottenfraßartige“ oder permeative Spongiosa- und/ oder Kortikalisdefekte charakterisiert.

Das Lodwick-Grading muss man systematisch lernen und üben, um die Befunde richtig interpretieren zu können.
Schwierige Befunde. Manche benignen Läsionen ändern durch eine Traumatisierung ihre Morphologie, z. B. eine traumatisierte fibröse Dysplasie. Durch das Trauma wird Knochen in kurzer Zeit abgebaut, und eine vorher im Lodwick-Grad IA befindliche Läsion wandelt sich dadurch in einen Defekt mit unscharfen Rändern und manchmal auch in ein mottenfraßartiges Aussehen um.

Eine weitere Schwierigkeit bei der Interpretation von osteolytischen Läsionen in Kombination mit einer Spontanfraktur liegt in der Möglichkeit der Verwechslung von Kallus mit Tumorosteoid, das z. B. durch ein spontan frakturiertes Osteosarkom primärer und sekundärer Art produziert wird. Am Rand mancher gutartigen Läsion (z. B. fibroossäre Läsion am Schenkelhals) kann sich ein maligner Prozess wie ein Fibrosarkom entwickeln und Anlass zu einer Spontanfraktur sein. Solche Fälle sind immer außerordentlich schwierig zu diagnostizieren.

Frakturrisiko. Radiologisch besteht eine Spontanfrakturgefährdung bei:

- jeder Osteolyse im Schenkelhals

- allen osteolytischen Veränderungen im Wirbelkörper, besonders in den Anhangsgebilden

- einer Zerstörung von mindestens $1 / 3$ der Kortikalis in großen Röhrenknochen

- Plasmozytom und Bronchialkarzinom, da die Tumorzellen dieser Läsionen Osteoklasten stimulierende Faktoren bilden können

Prognosefaktoren. Die Heilung einer Spontanfraktur hängt meist, wie bei je-
Lodwick-Grading. Es sollte jedem, der sich mit der Interpretation von Röntgenbildern befasst, bekannt sein. Dabei han- 
Trauma Berufskrankh 2008 · 10[Suppl 2]:165-166

DOI 10.1007/s10039-008-1363-3

(c) Springer Medizin Verlag 2008

\section{J. Freyschmidt \\ Die pathologische Fraktur aus Sicht des Radiologen}

\section{Zusammenfassung}

Die konventionelle Einteilung von Frakturen wird der Klinik und dem radiologischen Befund nicht ganz gerecht, besser geeignet ist eine logische Klassifikation, die generell eine traumatische von einer pathologischen Fraktur unterscheidet. Auf eine Spontanfraktur hinweisend sind Schmerzen schon vor dem Bruch, Vorgeschichte des Patienten, mottenfraßartiges Aussehen der Ränder einer Spontanfraktur, einem Lodwick-Grad II-III (s. unten) entsprechend, und Fehlen von $>50 \%$ der Zirkumferenz. Neben Röntgenaufnahmen können bei der Diagnose von Spontanfrakturen auch CT oder MRT zum Einsatz kommen, da damit prognostisch wichtige Faktoren - das Ausmaß der Zerstörung der Vaskularisation und der Knochennerven sowie das eines
Begleithämatoms - bestimmt werden können. Zu Insuffizienzfrakturen kommt es v. a. bei Osteoporose oder Osteomalazie. Sie können bei guter Aufnahmetechnik eindeutig aus dem Röntgenbild diagnostiziert werden. Die Betroffenen sollten postoperativ unbedingt in eine Institution überwiesen werden, in welcher die Grunderkrankungen weiter diagnostiziert und therapiert werden können, weshalb in den Arztbriefen grundsätzlich auf die Ursache der Insuffizienzfraktur hingewiesen werden sollte.

\section{Schlüsselwörter}

Pathologische Fraktur - Traumatische Fraktur . Klassifikation · Lodwick-Grad · Arztbrief

\section{Pathological fractures from a radiological point of view}

\section{Abstract}

The conventional classifiaction of fractures does not always take the clinical and radiological findings sufficiently into consideration; a logical classification distinguishing generally between traumatic and pathological fractures is more suitable. Indications of a spontaneous fracture include pain prior to fracture, patient history, moth-eaten appearance of the fracture borders, corresponding to Lodwick grade II--III, and bone circumference reduction of $>50 \%$. In addition to $X$ rays, CT or MRI can be helpful in the diagnosis of spontaneous fractures by establishing prognostically important factors, i.e., the extent of vascular and osseous nerve destruc- tion, as well as concomitant hematoma. Osteoporosis or osteomalacia are the most frequent causes of insufficiency fractures, which can be readily diagnosed on adequate $X$-ray images. Post-operatively, patients should be referred to a suitable institution for further diagnosis and therapy of the underlying disease. For this reason, doctor's letters should make explicit mention of the cause of insufficiency fracture.

\section{Keywords}

Pathological fracture - Traumatic fracture . Classification · Lodwick scale · Doctor's letter der traumatischen Fraktur, vom Ausmaß der Zerstörung der Vaskularisation und der Knochennerven ab. Letzteren kommt bei der Knochenheilung besondere Bedeutung zu, da die Nervenzellen offensichtlich neurotrophe Stoffe bilden, die die Zellteilung stimulieren. Des Weiteren wird die Heilung vom Ausmaß eines Begleithämatoms v. a. bei hochvaskularisierten Tumoren (z. B. Metastase eines Nierenzellkarzinoms) beeinflusst. Um beide Risikofaktoren besser definieren zu können, sind eine computertomographische Untersuchung ohne und mit Kontrastmittel oder auch eine MRT-Untersuchung durchaus sinnvoll.

Insuffizienzfrakturen. Sie werden heute besonders bei älteren Frauen mit Osteoporose oder Osteomalazie beobachtet, sie können bei guter Aufnahmetechnik eindeutig aus dem Röntgenbild diagnostiziert werden. Sie müssen häufig chirurgisch versorgt werden (z. B. distale Radiusfraktur, Schenkelhalsfrakturen). Die Patienten sollten postoperativ in eine Institution überwiesen werden, in welcher die Grunderkrankungen weiter diagnostiziert und therapiert werden können. Deshalb sollte in den Arztbriefen unbedingt auf die Ursache einer Insuffizienzfraktur hingewiesen und nicht nur die Verletzung und deren Versorgung festgestellt werden. Sonst kommen diese Patienten nach nicht allzu langer Zeit mit der - vermeidbaren nächsten Fraktur zur Aufnahme.

\section{Korrespondenzadresse}

Prof. Dr. J. Freyschmidt

Beratungsstelle und Referenzzentrum für Osteoradiologie, Klinikum Bremen Mitte, Friedrich-Karl-Straße 55, 27177 Bremen freyschmidt@radiologie-freyschmidt.de

Interessenkonflikt. Der korrespondierende Autor gibt an, dass kein Interessenkonflikt besteht.

\section{Literatur}

1. Freyschmidt J (2008) Skeletterkrankungen - klinisch-radiologische Diagnose und Differentialdiagnose, 3. Aufl. Springer, Berlin Heidelberg New York 